Anaesthesist 2015 - 64:901-902

DOI 10.1007/s00101-015-0122-7

Online publiziert: 2. Dezember 2015

(c) Springer-Verlag Berlin Heidelberg 2015

CrossMark

\author{
H. Andel \\ Universitätsklinik für Anästhesie, allgemeine Intensivmedizin und Schmerztherapie, \\ Medizinuniversität Wien, Wien, Österreich
}

\title{
Gedanken zur Fehlerkultur
}

Personen als Verursacher zu beschuldigen („name-blame-shame culture“).

\section{Probleme der Organisationsstruktur}

Inzwischen weiß man aber, dass Fehlerursachen praktisch nie allein auf handelnde Person, sondern in aller Regel auf das Arbeitsumfeld zurückzuführen sind. Unabhängig von handelnden Personen sind diese in der Organisationsstruktur verankert. Eine Vielzahl von Untersuchungen aus der Hochrisikoindustrie wie beispielsweise Luftfahrt oder Kernkraft zeigt, dass ein ausschließlich personenfixierter Ansatz auch in Hinblick auf die Vermeidung zukünftiger Fehler grundfalsch ist. Somit ist ein proaktiver Ansatz, der nach aufgetretenen Fehlern eine personenunabhängige Erforschung der Fehlerursachen vorsieht („Root-Cause- Analyse“), aber auch nach potentiellen Fehlermöglichkeiten bereits vor ihrer Verwirklichung sucht wesentlich effektiver.

Gerade im perioperativen Bereich wird die Bildung einer solchen Kultur des Risikobewusstseins durch die derzeit vorhandenen strukturellen Gegebenheiten erschwert. Alle Mitglieder der unterschiedlichen Krankenhausberufsgruppen lernen schon sehr früh, dass es nicht immer gelingt, ein wirklich zufriedenstellendes Behandlungsergebnis zu erreichen. Der Zustand eines Patienten kann sich, trotz Ausschöpfung aller Möglichkeiten, auch verschlechtern. Gerade im Bereich der Anästhesiologie fehlt vielen Mitarbeitenden der Überblick über den weiteren Krankheitsverlauf der Patienten. Diese Tatsache spielt eine wichtige Rolle bei der Beurteilung der erbrachten Leistungen aber auch bei Fehlleistungen. Im Übrigen ist es für den Einzelnen häufig nicht möglich, die tatsächliche Ursache für die Ver- schlechterung eines Patientenzustandes zu erkennen. Durch die Diskontinuität der Patientenbehandlung bzw. fehlenden abteilungsübergreifenden Prozessverantwortlichkeiten kann es geschehen, dass aufgetretene Fehler unbeachtet bleiben und ungünstige Verläufe als schicksalhaft angenommen werden.

Neben den technischen Aspekten, die sich in erster Linie mit Methoden der Risikoerkennung, Risikobewertung und Risikominimierung, sowie die Messung der Effektivität beschäftigen, steht die Schaffung einer Unternehmenskultur im Vordergrund. Wie bereits oben angemerkt ist traditionell gerade im ärztlichen Bereich das Eingestehen von Fehlern nicht selbstverständlich. Ein solches Umfeld verhindert den proaktiven Umgang mit Risiken der Patientenversorgung nachhaltig.

Dem gegenüber steht der Zugang der „Root-Cause-Analyse“, wie er von der „Joint Commission“ bereits seit Jahren gefordert wird [2].

Hier werden „sentinel events“ definiert („A sentinel event is an unanticipated occurrence involving death or major permanent loss of function unrelated to the natural course of the patient's illness or underlying condition"). Jeder einzelne „sentinel event“ muss der Joint Commission gemeldet werden und es muss verpflichtend eine Ursachenanalyse durchgeführt werden. Der wesentliche Unterschied zur „Name-Blame-ShameKultur" liegt hierbei in einem systemanalytischen Ansatz, wie er im London Protocol beschrieben wird [3].

Die tägliche Patientenversorgung besteht aus vielen einzelnen, teilweise ineinander greifenden Maßnahmen unterschiedlichster Intensität. Je kränker Patienten sind, desto mehr Risikofaktoren für unerwünschte Zwischenfälle bringen sie mit sich. Gleichzeitig werden solche klärungspflicht Genüge getan zu haben, wenn es gelungen ist, eine der beteiligten 
Patienten mit den Folgen solcher verwirklichten Risiken gesundheitlich deutlich mehr belastet als Patienten, die von vorne weg ein geringeres Behandlungsrisiko aufweisen. Je schlechter die Ausgangslage der Patienten ist desto belastender und gefährlicher sind im Allgemeinen die notwendigen Eingriffe, um Krankheiten und Verletzungen zu erkennen, zu heilen oder zu lindern. Naturgemäß ist ein höheres Risiko auch mit einer höheren Komplikationsrate behaftet. Gerade die zusätzliche Schädigung von Patienten stellt für Krankenhausmitarbeiter ein stark belastendes Geschehnis dar. Neben den Selbstvorwürfen können zusätzlich berufliche sowie straf- und zivilrechtliche Konsequenzen drohen („second victim“). Folglich ist es nicht einfach, sich selbst oder anderen gegenüber aufgetretene Fehler einzugestehen. Entscheidend für die Entwicklung einer Sicherheitskultur ist deshalb die systematische Entwicklung von Maßnahmen zur Stützung von Mitarbeitenden, die in der Behandlung von Patienten, bei denen sich ein Risiko verwirklicht hat, involviert waren [4].

\section{Der Mitarbeiter muss geschützt werden}

Nur wenn es gelingt, eine Fehlerkultur $\mathrm{zu}$ etablieren, die den einzelnen Mitarbeitenden schützt bzw. unterstützt, wird die Implementierung von Methoden zum Risikomanagement eine maximale Wirkung erzielen können.

Somit stellt neben der (zwar Kosten verursachenden) Einführung von Risikomanagementprozessen die Entwicklung einer Kultur, die einen vom Einzelnen losgelösten Umgang mit Fehlern ermöglicht, den entscheidenden Schritt auf dem Weg zur Verbesserung der Patientensicherheit dar.

Ein weiteres, nicht unwesentliches Risiko stellt die Erhöhung der Behandlungsdichte als Folge der Ausnützung von Einsparungspotentialen mit Hilfe externen Beratungsfirmen zur Prozessoptimierungen dar. Insbesondere die gleichzeitige Zunahme des Dokumentationsaufwandes und steigende Patientenzahlen führen $\mathrm{zu}$ einer $\mathrm{Zu}$ nahme der Personalbelastung. Dadurch steigt auch das Risiko, dass Fehler durch Überbelastung entstehen.

Erfahrungsgemäß muss in einem Krankenhausbetrieb immer mit Verzögerungen durch organisatorische, kommunikative oder technische Probleme, mit individuellen Unterschieden bei den Patienten, aber auch den behandelnden Ärzten gerechnet werden. Eine komplette Verplanung des zur Verfügung stehenden Zeitrahmens wird deshalb regelmäßig Grenzsituationen provozieren. Ebenso steigt bei vielen Mitarbeitenden die Bereitschaft, sich auch für minimale Zeitvorteile auf riskante Handlungsweisen einzulassen. Dieses Verhalten ist besonders stark ausgeprägt, wenn mehrere Berufsgruppen und Disziplinen direkt zusammenwirken. Hier verleitet die Erwartungshaltung Anderer dazu, unnötige Risiken einzugehen.

In diesem Zusammenhang ist auch $\mathrm{zu}$ berücksichtigen, dass eine vollständige Auslastung der vorhandenen Ressourcen verhindert, dass sich die Mitarbeitenden kreativ an der Verbesserung ihres Arbeitsplatzes und damit auch an der Minderung von Risiken beteiligen können.

Vor diesem Hintergrund kommt der Entwicklung eines funktionierenden, synergistischen Risikomanagementkonzeptes, das sich mit den jeweiligen Stärken und Schwächen der einzelnen Elemente auseinandersetzt und diese berücksichtigt eine entscheidende Bedeutung in der Bewältigung des perioperativen Risikos zu.

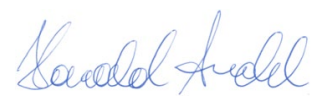

H. Andel

\section{Korrespondenzadresse}

Ao. Univ. Prof. Dr. H. Andel
MSc, MLS
Universitätsklinik für Anästhesie,
allgemeine Intensivmedizin und
Schmerztherapie,
Medizinuniversität Wien
Währinger Gürtel 18-20
1090 Wien
Harald.andel@meduniwien.ac.at

\section{Literatur}

1. Neuhaus C, Röhrig R, Hofmann G, Klemm S, Neuhaus $S$, Hofer S, Thalheimer M, Weigand M, Lichtenstern C (2015) Patientensicherheit in der Anästhesie - multimodale Strategien für die perioperative Versorgung. Anästhesist. doi:10.1007/ s00101-015-0115-6

2. http://www.jointcommission.org/about_us/ about_the_joint_commission_main.aspx. Zugegriffen: 24. Nov. 2015

3. https://www1.imperial.ac.uk/resources/ 3AD8B321-0916-47D2-A196-1A993E36D0B5/ londonprotocoldeutsch.pdf. Zugegriffen: 24. Nov. 2015

4. Wu AW, Boyle DJ, Wallace G, Mazor KM (2013) Disclosure of adverse events in the United States and Canada: an update, and a proposed framework for improvement. J Public Health Res 2(3):e32. doi:10.4081/jphr.2013.e32. eCollection 2013. http://www.ncbi.nlm.nih.gov/pmc/articles/ PMC4147741/pdf/jphr-2013-3-e32.pdf. Zugegriffen: 24. Nov. 2015 